\title{
A Calibration of the Wierzbicki-Xue Damage Model Using Charpy Test Results
}

\author{
Jong-Bong Kim ${ }^{1, a}$, Hyunho Shin ${ }^{2, b}$, Yo-Han Yoo ${ }^{3, c}$ \\ ${ }^{1}$ Department of Mechanical and Automotive Engineering, Seoul National University of Science and Technology, 232 Gongneung-ro, \\ Nowon-gu, Seoul, Republic of Korea \\ ${ }^{2}$ Department of Advanced Ceramic Materials Engineering, Gangneung-Wonju National University, 7 Jugheon-gil, Gangneung, Gangwon-do \\ 210-702, Republic of Korea \\ ${ }^{3}$ Agency for Defense Development, Daejeon, 305-600, Republic of Korea
}

\begin{abstract}
Damage models are frequently used to predict fractures in large deformation problems such as penetration of a projectile into a target. Though many damage models have been proposed so far, coefficients of each model have been provided for only a few materials. In this study, the coefficients of the Wierzbicki-Xue (2005) damage model for tungsten heavy alloy (DX2HCMF) are determined using the Charpy impact test. The Wierzbicki-Xue fracture criterion is implemented into NET3D code in which a node-split algorithm is built in. By comparing the energy absorbed in the Charpy test with the results of finite element analysis, the fracture model coefficients are determined.
\end{abstract}

\section{Introduction}

Various damage models have been proposed to analyze the fracture behavior of metals [1-8]. Gurson [1] modeled the damage criterion considering void nucleation, growth, and coalescence during fracture. Cockcroft and Latham [3] proposed a damage model that is a function of the principal stress and effective strain. Johnson and Cook [4] defined the critical fracture strain as a phenomenological function of the stress triaxiality, strain rate, and temperature. Wierzbicki et al. (2005) assumed that a fracture occurs when the accumulated equivalent plastic strain, modified by a function of the stress triaxiality and the deviatoric state parameter, reaches a critical value. Wierzbicki et al. [5] intensively reviewed the fracture prediction capability of several damage models by comparing their predictions with experimental results. Recently, Xue [6-7] included the effects of the pressure, Lode angle, and stress in the fracture criterion of Wierzbicki et al. [5]. Stoughton and Yoon [8] proposed a stress-based fracture and necking criterion; their fracture models were successful in predicting the fractures in a sheet metal forming process. Some of the above models were implemented in commercial finite element codes [5] such as ABAQUS [9] and LS-DYNA [10]. However, the model coefficients have been provided for only a few materials so far; Wierzbicki et al. [5] determined model parameters for aluminum 2024-T351 using several sets of experimental results. In this study, the Charpy impact test has been carried out for tungsten heavy alloy
(DX2HCMF) to calibrate the coefficients of the Wierzbicki-Xue [5] damage model. The energy absorbed in the Charpy test is measured. Finite element analysis is carried out for varying values of the damage model parameters until these values reasonably predict the experimentally measured absorption energy.

\section{Finite Element Analysis Model}

The finite element code NET3D [11-12], in which a node-split algorithm [11] is built-in, is used for numerical analysis. The fracture behavior of materials can be predicted using the node-split method. For the split criterion, the Wierzbicki-Xue damage model [5] is used; this model is defined as follows.

$$
\begin{aligned}
& \bar{\varepsilon}_{f}=C_{1} \exp \left(-C_{2} \eta\right)-\left[C_{1} \exp \left(-C_{2} \eta\right)-C_{3} \exp \left(-C_{4} \eta\right)\right]\left(1-\xi^{1 / n}\right)^{n} \\
& \eta=\sigma_{m} / \bar{\sigma} \\
& \xi=\frac{27 J_{3}}{2 \bar{\sigma}^{3}}=\frac{27 \sigma_{1} \sigma_{2} \sigma_{3}}{2 \bar{\sigma}^{3}}
\end{aligned}
$$

where is the fracture strain, is the stress triaxiality, is the third invariant of the deviatoric stress, and $\mathrm{C}_{1}, \mathrm{C}_{2}$, $\mathrm{C}_{3}, \mathrm{C}_{4}$, and $\mathrm{n}$ are model parameters to be determined. In this model, the damage is defined as,

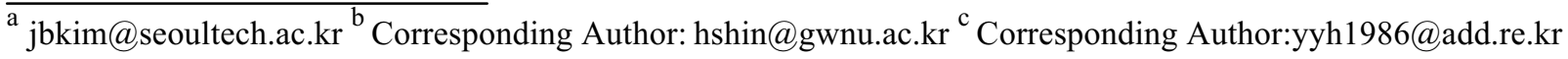



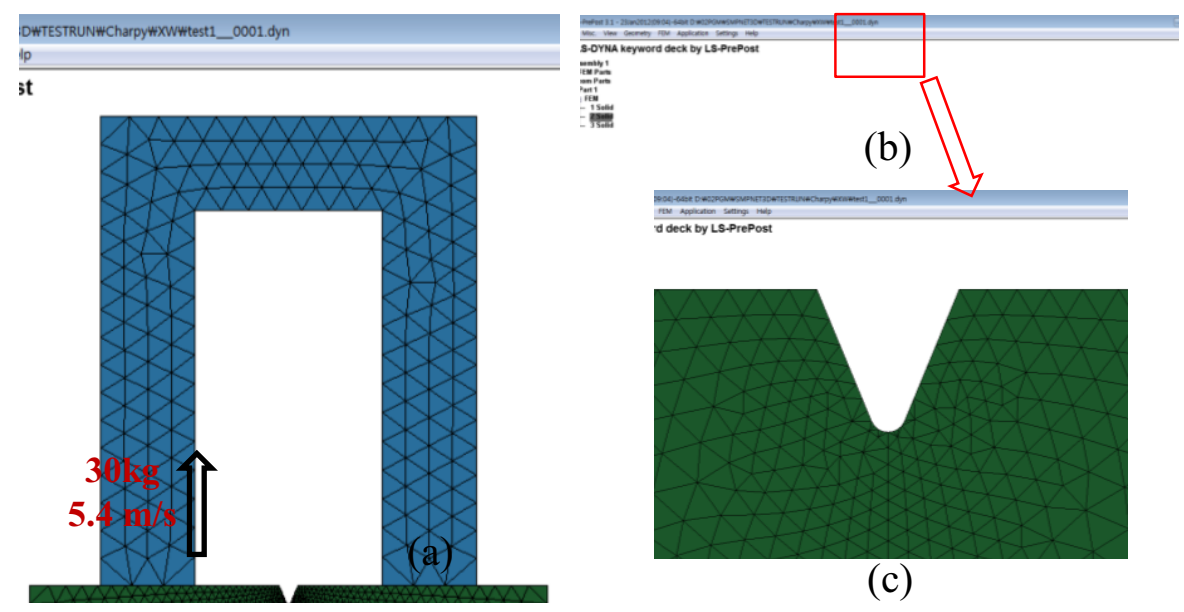

(c)

Figure. 1. Finite element analysis model for Charpy impact test

Table 1. Parameters of the Johnson-Cook constitutive model (DX2HCMF)

\begin{tabular}{ccccccc}
\hline Parameters & $A[\mathrm{MPa}]$ & $B[\mathrm{MPa}]$ & $C$ & $n$ & $m$ & $T_{\mathrm{m}}\left[{ }^{\circ} \mathrm{C}\right]$ \\
\hline Value & 1085 & 1900 & 0.032 & 0.390 & 1.0 & 1,520 \\
\hline
\end{tabular}

$$
D=\int \frac{d \bar{\varepsilon}}{\bar{\varepsilon}_{f}(\eta, \xi)}
$$

When the value of the damage $(D)$ reaches 1.0, a fracture takes place. In this analysis, an element face is divided into two faces when both of the elements sharing the face have damage values greater than 0.98 . In contrast to the case of the element erosion method, after the fracture, the split element can bear a compressive load.

Fig. 1 illustrates the analysis model; this model has the same dimensions as those of the experiment (the Charpy impact test). Analysis was carried out in threedimensions. For accurate analysis, the notch region was discretized using smaller elements. The Johnson-Cook model was employed as the constitutive model; its parameters for tungsten heavy alloy (DX2HCMF) are shown in Table 1.

\section{Results and discussion}

Fig. 2 shows the fracture shapes at several time intervals. No fracture takes place until $50 \mathrm{~ms}$; it occurs at $50 \mathrm{~ms}$ at the notch region marked by the arrow (Fig. 2(c)). Many small fragments are observed thereafter, as can be seen in Figs. 2(d) (f). The element faces newly generated by the fracture were assigned to the contact face for contact treatment. Therefore, no penetration was allowed for the contacted nodes and edges. The residual velocity of the impactor was monitored and the absorbed energy was calculated using the initial and residual velocities. For a comparison with the analysis result, three Charpy tests were carried out. The measured absorbed energies in the three tests were $14.5,16.4$, and $11.8 \mathrm{~J}$ and the averaged value was $14.2 \mathrm{~J}$. Through separate preliminary analyses, $\mathrm{C}_{1}$ and $\mathrm{C}_{2}$ values were fixed at 0.28 and 5.56, respectively. Examples of the simulated values of the loss of kinematic energy in this study are shown in Fig. 3 (for varying $\mathrm{C}_{3}$ values when $\mathrm{C}_{4}$ values were 0.7 and 1.2). The kinematic energy losses before the fracture were almost the same for all of the considered cases. The initiation and resistance to fracture were influenced by the model parameters. Among the simulation cases, the parameter set of $\mathrm{C}_{1}=0.28, \mathrm{C}_{2}=5.56, \mathrm{C}_{3}=0.1, \mathrm{C}_{4}=1.2$, and $\mathrm{n}=0.5$ mose closely predicted the experimentally determined absorbed energy. 

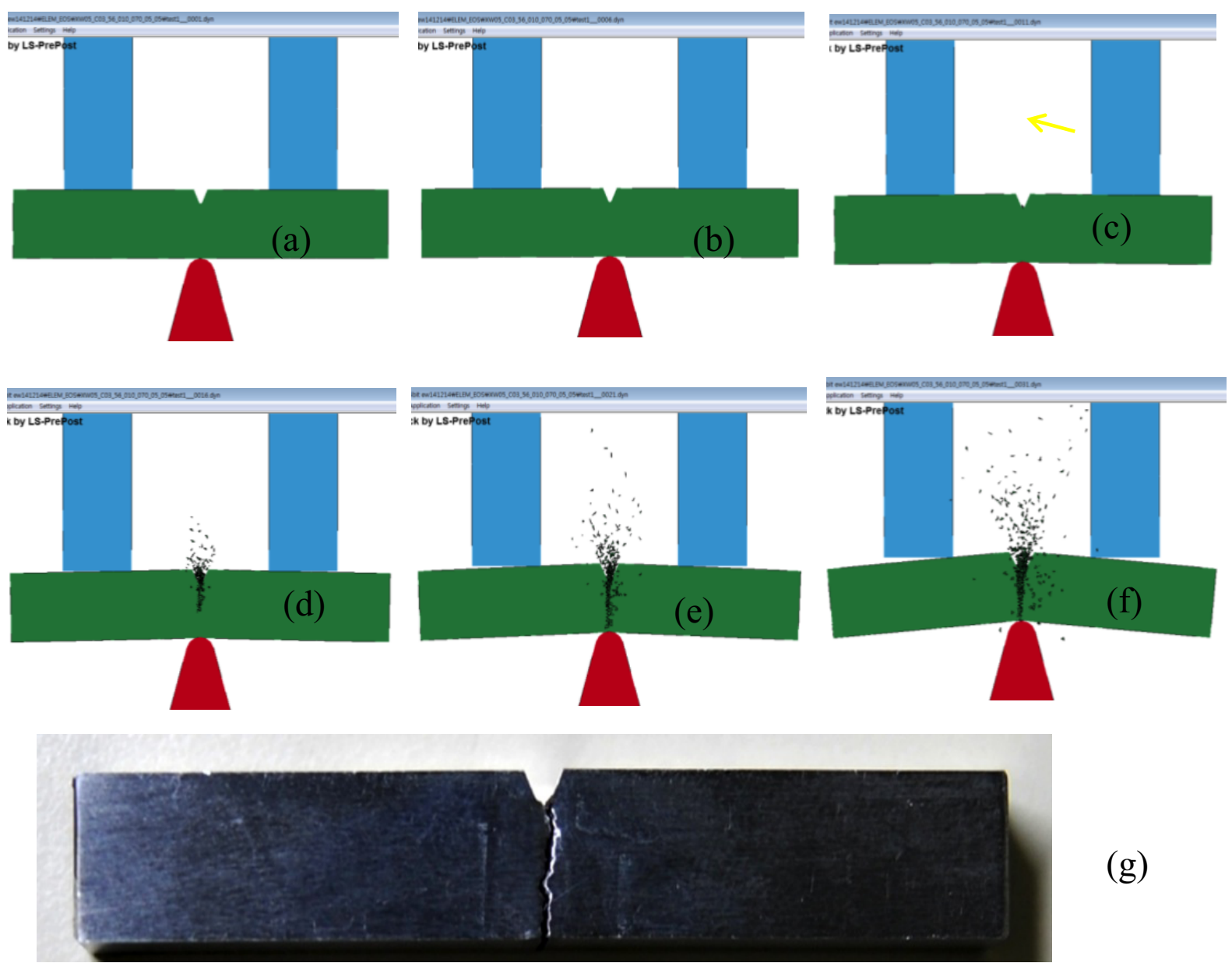

(g)

Figure. 2. Fracture shapes at (a) $0 \mu \mathrm{s}$, (b) $25 \mu \mathrm{s}$, (c) $50 \mu \mathrm{s}$, (d) $75 \mu \mathrm{s}$, (e) $100 \mu \mathrm{s}$, and (f) $150 \mu \mathrm{s}\left(C_{1}=0.28, C_{2}=5.56, C_{3}=0.1, C_{4}=0.7\right.$, $n=0.5)$, and (g) the experimental specimen after the fracture
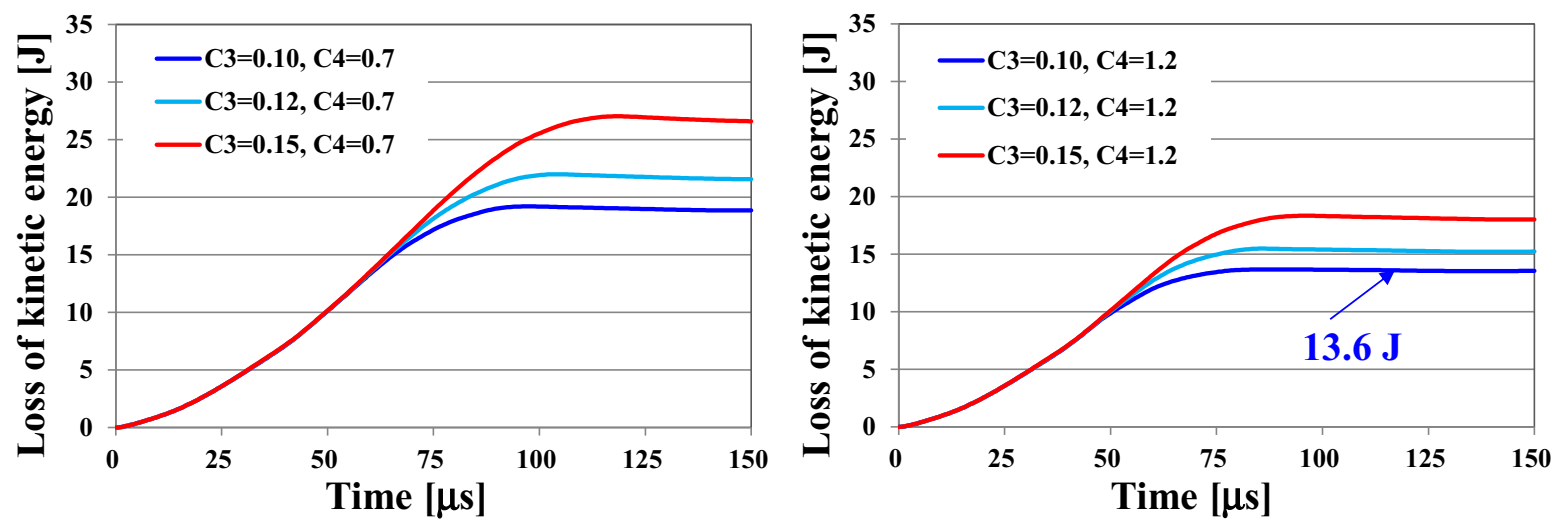

Figure. 3. Loss of kinematic energy history for various fracture model parameters $\left(C_{l}=0.28, C_{2}=5.56, n=0.5\right)$.

\section{Summary}

The Wierzbicki-Xue damage model was implemented to a finite element code (NET3D). Internal element faces were separated when the damage value was greater than 0.98. Finite element analysis was carried out for varying damage model parameter sets. The loss of kinematic energy of the impactor was calculated by FE analysis and compared with that obtained by the experiment. The model parameters that most accurately predicted the experimentally obtained absorbed energy were: $\mathrm{C}_{1}=0.28$, $\mathrm{C}_{2}=5.56, \mathrm{C}_{3}=0.1, \mathrm{C}_{4}=1.2$, and $\mathrm{n}=0.5$.

\section{Acknowledgments}

This study was supported by the Research Program funded by the Seoul National University of Science and Technology. 


\section{References}

1. A.L. Gurson, Plastic flow and fracture behaviour of ductile materials incorporating void nucleation, growth and coalescence, PhD Diss., Brown University, 1975.

2. V. Tvergaard, On localization in ductile materials containing spherical voids, Int. J. Fract. 18 (1982) 237-252.

3. M.G. Cockcroft, D.J. Latham, Ductility and the workability of metals, J. of the Inst. of Metals 96 (1968) 33-39.

4. G.R. Johnson, W.H. Cook, Fracture characteristics of three metals subjected to various strains, strain rates, temperatures and pressures, Eng. Fract. Mech. 21 (1985) 31-48.

5. T. Wierzbicki, Y. Bao, Y.-W. Lee, Y. Bai, Calibration and evaluation of seven fracture models, . Int. J. mech. Sci. 47 (2005) 719-743.
6. L. Xue, Damage accumulation and fracture initiation in uncracked ductile solids subject to triaxial loading, Int. J. Solids Struct. 44 (2007) 5163-5181.

7. L. Xue, Stress based fracture envelope for damage plastic solids, Eng. Fract. Mech. 76 (2009) 419-438.

8. T.B. Stoughton, J.W. Yoon, A new approach for failure criterion for sheet metals. International Journal of Plasticity 27 (2010)440-459.

9. ABAQUS Inc., ABAQUS Theory manual, version 6.12, 2012.

10. Livermore software technology co., LS-Dyna manual (http://www.dynasupport.com/manuals), version 971, 2015.

11. M. Lee, E.Y. Kim, Y.-H. Yoo, Simulation of high speed impact into ceramic composite systems using cohesive-law fracture model, Int. J. Impact Eng. 35 (2008) 1636-1641.

12. Y.-H. Yoo, H. Shin, Protection capability of dual flying plates against obliquely impacting long-rod penetrators, Int. J. Impact Eng. 30 (2004) 55-68. 